\title{
A Neural Network Model for Team Viability
}

\author{
Isabel Dórdio Dimas ${ }^{1,2}$, Humberto Rocha ${ }^{3,4}$, \\ Teresa Rebelo ${ }^{5,6}$, and Paulo Renato Lourenço ${ }^{5,6}$ \\ 1 ESTGA, Universidade de Aveiro, \\ 3750 - 127 Águeda, Portugal \\ 2 GOVCOPP, Universidade de Aveiro, \\ 3810-193 Aveiro, Portugal \\ 3 CeBER and FEUC, Universidade de Coimbra, \\ 3004-512 Coimbra, Portugal \\ 4 INESC-Coimbra, \\ 3030-290 Coimbra, Portugal \\ 5 IPCDVS, Universidade de Coimbra, \\ 3001-802 Coimbra, Portugal \\ ${ }^{6}$ FPCEUC, Universidade de Coimbra, \\ 3000-115, Coimbra, Portugal \\ idimas@ua.pt, hrocha@mat.uc.pt, terebelo@fpce.uc.pt, \\ prenato@fpce.uc.pt
}

\begin{abstract}
Team effectiveness has been the focus of numerous studies since teams play an increasingly decisive role in modern organizations. In the present paper, our attention is centered on team viability, which is one dimension of team effectiveness. Given the challenges that actual teams face today, exploring the conditions and processes that enhance the capacity of teams to adapt and continue to work together is a fundamental research path to pursue. In this study, team psychological capital and team learning were considered as antecedents of team viability. The relationships that team psychological capital and team learning establish with team viability were explored as accurately as possible. Typically, these relationships are assumed to be linear as multivariate linear models are often used. However, these linear models fail to explain possible nonlinear relations between variables, expected to exist in dynamic systems as teams. Adopting computational modeling strategies in the context of organizational psychology has become more common. In this paper, radial basis function models and neural networks were used to study the complex relationships between team psychological capital, team learning and team viability.
\end{abstract}

Keywords: Team viability, Radial basis functions, Neural Networks

\section{Introduction}

Teams are, nowadays, a fundamental cornerstone of organizations. Since teams are created with the aim of generating value, understanding the conditions that contribute to team performance has been one of the major focus 
of research (e.g., [16]). However, although performance is a fundamental team effectiveness dimension, effectiveness is much more than performance. In fact, the literature is consensual about the need to consider different criteria to assess effectiveness [17/21. We will focus our attention on team viability, which is in line with one of the dimensions of the Hackman's three dimensional effectiveness approach $17 / 18$. Team viability can be defined as the team's capacity to adapt to internal and external changes as well as the extent to which team members are able to continue to work together in the future [39. Given the challenges that actual teams face today, such as working with new technology or the need to constantly adapt to market fluctuations and changes, exploring the conditions and processes that enhance the capacity of the team to adapt and continue to work together is a fundamental path to pursue. In this study, team psychological capital and team learning will be considered as antecedents of team viability.

The term psychological capital can be defined as a positive, individual psychological state, or, in other words, a personal characteristic that can be measured and developed 22/24. Luthans and colleagues 22/24, using a number of key criteria, identified four main psychological resources that formed the higher-order concept of psychological capital: self-efficacy, hope, optimism, and resilience. Self-efficacy, based on Bandura's social cognitive theory [4, refers to the individual belief in his or her ability to successfully execute a specific task 38. Hope is characterized by two dimensions: will power, i.e., the drive and determination to attain a goal; way power thinking, i.e., the ability to plan alternative ways for attaining a desired goal 23. Optimism is defined as the individuals expectancy of positive outcomes and integrates both realism and flexibility [41. Finally, resilience is the ability to withstand and recover from challenges, stressful events or any other threat to well-being [43].

The relationship between individual-level psychological capital and effectiveness, namely, performance, has been investigated and established by a large number of studies 2 241. Results are, however, less consistent when we consider some psychological resources separately, namely self-efficacy. For instance, concerning the relationship between self-efficacy and performance, whereas some studies, focusing on a socio-cognitive theory of self-regulation [4], found a positive relationship between self-efficacy and performance, others report that selfefficacy might lead to overconfidence, increasing, in consequence, the chance of committing errors during the tasks and affecting performance negatively 42 .

The research developed until this moment almost never focused psychological capital as a team level phenomenon [30. Teams have a major influence on the perceptions, decisions, beliefs and emotions of individuals and as a result of the interaction of knowledge between team members, shared mental models are created [26], which might lead to the development of a collective psychological capital [20]. Studies that focused psychological capital as a collective phenomenon, although scarce, point to a positive influence of this collective psychological state on team outcomes (e.g., [20]27]).

Concerning team learning, this construct can be conceptualized as both a process and an outcome [12]: the former concerns the group member behaviors 
through the interaction processes, and the latter is the manifested outcome or result that emerges as a collective property of the team. In the present research our focus will be on team learning behaviors.

In line with Edmondson [14, team learning behaviors can be conceived as a continuous process of reflection and action, characterized by five fundamental behaviors: seeking feedback, both internally and externally, in order to measure group's effectiveness and to investigate possible improvements; exploring through the sharing of knowledge and perspectives, as well as through constructively managing different opinions; experimenting collectively new ways of achieving goals; reflecting on past achievements and on future aims and goals; and discussing errors and unexpected outcomes collectively and exploring ways to prevent them.

Previous research presented team learning as a crucial process of adaptation of teams to their environment and highlighted its importance to team effectiveness (e.g., $8[14[15])$. Nevertheless, some studies suggest that team learning may act as a double-edge sword. Bunderson and Sutcliffe [6], for instance, found that too much emphasis on learning can compromise efficiency because detract the team from results, and this is particular salient for teams that have been performing well. In line with these findings, we have found, previously, increasing trends on team effectiveness up to a certain threshold of team learning, followed by a deflation for the highest values of this variable [13. Taken together, these results highlight that more team learning is not always better and claim for more studies that support the non-linear relationship between team learning and team outcomes.

Recently, the importance of adopting computational modeling strategies in the context of organizational psychology was stressed by Cortina et al. 11. As outlined by Hanges et al. [19], the adoption of more complex designs, such as radial basis functions neural networks, are useful for modeling nonlinear behavior as produced by dynamic systems, such as, teams. In this paper, radial basis functions (RBF) regression and RBF neural networks are used to study the complex relationships between team psychological capital, team learning and team viability. RBF regression has been successfully applied in different contexts, including aeronautics [32]33] or radiotherapy [35|36]. RBF models proved to mimic well unknown responses providing reliable surrogates that can be used either for prediction or to extract relationships between variables. On the other hand, neural networks have been widely used for deep learning with large data sets [37. Incorporating RBF in the neural network learning process might enhance the extraction of nonlinear relationships between explanatory variables and response variable(s).

\section{Materials and Methods}

\subsection{Sample}

A quantitative study with a cross-sectional design was conducted, between November 2016 and January 2017, in which we surveyed teams from the Por- 
tuguese organizational setting. In order to be selected for the present study, teams must meet the following criteria: teams must consist of at least three members (1), who are perceived by themselves and others as a team (2), and who interact regularly and interdependently to accomplish a common goal (3) [10]. Different kind of measures were administered to team members and their respective leaders. Team members were surveyed about team psychological capital. Team leaders were surveyed about team learning and team viability. The use of different data sources contribute both to obtain a broader diagnose about the team and to prevent the problems related with common method variance. Two different strategies were used in data collection. In the majority of the organizations, data collection occurred in the organization facilities, with the physical presence of trained research assistants. When this strategy was not possible to implement, the questionnaires were filled in online via an electronic platform, with the link being provided to the participants. In both cases, the participation in the study was voluntary and was clarified on the front page of the survey that only aggregated data would be reported and that all identifying information would be removed. Besides, informed consent was obtained from all participants.

Surveys were administered to 452 members and to 104 leaders of 104 workgroups from 66 Portuguese organizations. After eliminating from the sample the teams where less than $50 \%$ of members answered the questionnaire and also the questionnaires where more than $10 \%$ of the answers were missing [5], the sample remained with 82 teams (353 members and 82 leaders) from 57 organizations, the majority from services sector $(73 \%)$. Team size ranged from three to 18 members, with an average of six members $(\mathrm{SD}=3.55)$.

\subsection{Measures}

To measure team psychological capital we adapted to the Portuguese language the scale Psychological Capital Questionnaire (PCQ) developed by Luthans at al. 25. All items were reworded to reflect the group, rather than the individual, as the referent. This scale is composed of 24 items that assess the four dimensions of psychological capital (six items per dimension) that are measured on a 6-point Likert type scale from 1 (strongly disagree) to 6 (strongly agree). Sample items for self-efficacy, hope, resilience and optimism are "We feel confident analyzing a long-term problem to find a solution", "At the present time, we are energetically pursuing our work goals", "We usually take stressful things at work in stride" and "I always look on the bright side of things regarding my job", respectively. An Exploratory Factorial Analysis (EFA) was conducted, returning a five-factor solution that explained $62.76 \%$ of the total variance. Since the fifth factor had no theoretical support because it was only composed of reverse items from different dimensions, these items were sequentially eliminated. Besides, items with loadings below .50, were also sequentially eliminated from the solution. The final solution retained 18 items organized in four factors that jointly explained $65.83 \%$. The Cronbach alpha for efficacy was .90, for hope was .85 , for resilience was .75 and for optimism was .80 . Since psychological capital 
has been consistently analyzed as a second-order factor (e.g., [9]) and also because the four psychological capital dimensions were highly correlated (bi-variate correlations varied from .54 to .73) we decided to conduct a Confirmatory Factor Analysis (CFA) with the retained four-factor structure obtained from EFA and with psychological capital as a second order factor. The fit indices obtained were acceptable $\left[\chi^{2}(131)=302.97, p<.001, \chi^{2} /\right.$ gl. $=2.31$, CFI $=.95$, RMSEA $=$ $.06]$ and, in consequence, we will consider, in the following analyses, the global score obtained from the average score of the four dimensions. A sample item is "Team members go out and get all the information they possibly can from others such as customers, or other parts of the organization".

To measure team learning we adapted to the Portuguese language the scale developed by [14. This scale is constituted by seven items that are measured on a 5-point scale from 1 (almost never happens) to 5 (almost always happens). An EFA was conducted that returned a two-factor solution. Since the original scale is constituted only by one dimension, we decided to drop off, sequentially the items from the second dimension (items 2, 4 and 6). After eliminating items 2 and 4, a one-factor solution was obtained. However, the communalities and loadings of items 1 and 6 were low. As the referred items were both related to obtaining feedback and were the reverse of each other, and since it was important to have at least one item related to feedback, we decided to eliminate item 1 and to maintain item 6 . Besides, this solution presented a higher reliability. The final solution was then composed of four items (3, 5, 6 and 7) that explained $53.3 \%$ of variance and presented a Cronbach alpha of .69.

To measure team viability we used the scale developed by Aubé and Rousseau [3], which was previously adapted to the Portuguese language by Albuquerque [1. The scale is constituted by four items that are measured on a 5-point scale from 1 (almost doesn't apply) to 5 (almost totally applies). Since the Cronbach alpha for this scale was .68, and since the exclusion of item 3 , which was the item with the content less related to the definition of the construct, increased the alpha to .73 , the referred item was dropped from the scale. A sample item is "The members of this team could work a long time together".

\subsection{Radial Basis Functions Models}

Radial basis functions can furnish response surfaces able to explore/explain the nonlinear relationships between different input or explanatory variables and output or response variable(s). Furthermore, RBFs are often used to predict the response of a variable given the value of explanatory variables. For any given set of data points, a RBF model (surface) can be calculated even for poorly distributed data points in a high dimensional space. However, the RBF surface landscape, i.e. the relationship between and beyond the data points, depends on the choice of the basis function. Some RBFs can provide desirable trends while other may exhibit undesirable trends. Numerical selection of the most appropriate $\mathrm{RBF}$ for the given data set is advisable instead of an a priori choice based on the literature [34. A brief description of RBF model calculation is provided next. 


\section{RBF interpolation problems}

Let $y(\mathbf{x})$ denote the response for a given data point $\mathbf{x}$ (of $n$ components) such that the value of $y$ is only known at a finite set of $N$ input data points $\mathbf{x}^{1}, \ldots, \mathbf{x}^{N}$, i.e., only $y\left(\mathbf{x}^{k}\right)(k=1, \ldots, N)$ are known. A RBF interpolation model $h(\mathbf{x})$ can be generically represented as

$$
h(\mathbf{x})=\sum_{j=1}^{N} \alpha_{j} \varphi\left(\left\|\mathbf{x}-\mathbf{x}^{j}\right\|\right)
$$

where $\varphi(x)$ is the selected RBF, $\alpha_{j}$ are the coefficients determined by the interpolation equations $h\left(\mathbf{x}^{k}\right)=y\left(\mathbf{x}^{k}\right)(k=1, \ldots, N),\left\|\mathbf{x}-\mathbf{x}^{j}\right\|$ corresponds to the

parameterized distance between $\mathbf{x}$ and $\mathbf{x}^{j},\left\|\mathbf{x}-\mathbf{x}^{j}\right\|=\sqrt{\sum_{i=1}^{n}\left|\theta_{i}\right|\left(x_{i}-x_{i}^{j}\right)^{2}}$, and $\theta_{1}, \ldots, \theta_{n}$ are scalars 34. Coefficients $\alpha_{1}, \ldots, \alpha_{N}$ in Eq. (1) are computed for fixed parameters $\theta_{i}$ using the interpolation equations of the following linear system:

$$
\sum_{j=1}^{N} \alpha_{j} \varphi\left(\left\|\mathbf{x}^{k}-\mathbf{x}^{j}\right\|\right)=y\left(\mathbf{x}^{k}\right), \quad \text { for } k=1, \ldots, N .
$$

Multiquadric, $\varphi(x)=\sqrt{1+x^{2}}$, thin plate spline, $\varphi(x)=x^{2} \ln x$, cubic spline, $\varphi(x)=x^{3}$, and Gaussian, $\varphi(x)=\exp \left(-\mathrm{x}^{2}\right)$, are examples of RBFs that are commonly used used to model linear, almost quadratic and cubic growth rates, as well as exponential decay of the response, respectively [31.

Calculation of the RBF model $h(\mathbf{x})$ in Eq. (1) requires the selection of a RBF $\varphi(x)$ and the choice of model parameters $\theta_{1}, \ldots, \theta_{n}$. While selection of the most appropriate $\mathrm{RBF}$ for the given data set can be done iteratively by testing the different possible choices of $\varphi(x)$, there is an infinite number of possible choices for $\theta_{1}, \ldots, \theta_{n}$. For different fixed sets of model parameters $\theta_{1}, \ldots, \theta_{n}$, distinct models with different behaviors between data points are calculated for a given selection of $\varphi(x)$. Cross-validation (CV) can be used for model parameter tuning leading to models with enhanced prediction capability [40. Furthermore, the most appropriate basis function $\varphi(x)$ can be numerically computed using prediction accuracy (CV error) as main criterion. The leave-one-out $\mathrm{CV}$ procedure can be used in model parameter tuning for RBF interpolation [34:

\section{Algorithm 1 (Leave-one-out cross-validation for RBF interpolation).}

1. Fix a set of model parameters $\theta_{1}, \ldots, \theta_{n}$.

2. For $j=1, \ldots, N$, construct the RBF model $h_{-j}(\mathbf{x})$ of the data points $\left(\mathbf{x}^{k}, y\left(\mathbf{x}^{k}\right)\right)$ for $1 \leq k \leq N, k \neq j$.

3. Set prediction error as the following CV root mean square error:

$$
E^{C V}\left(\theta_{1}, \ldots, \theta_{n}\right)=\sqrt{\frac{1}{N} \sum_{j=1}^{N}\left(h_{-j}\left(\mathbf{x}^{j}\right)-y\left(\mathbf{x}^{j}\right)\right)^{2}} .
$$


The goal of model parameter tuning by $\mathrm{CV}$ is to find $\theta_{1}, \ldots, \theta_{n}$ that minimize the CV error, $E^{C V}\left(\theta_{1}, \ldots, \theta_{n}\right)$, so that the interpolation model has the highest prediction accuracy when CV error is the measure. Using different $\theta_{i}$ allows the model parameter tuning to scale each variable $x_{i}$ based on its significance in modeling the variance in the response, thus, has the benefit of implicit variable screening built in the model parameter tuning.

\subsection{RBF Neural Networks}

Neural Networks (NN) is a class of bio-inspired computer algorithms that attempt to mimic the human brain thinking process. This class of artificial intelligence methods have been widely used in machine learning, data mining or statistics. The main feature of $\mathrm{NN}$ is the ability to extract trends from large amounts of data whose patterns are difficult to perceive by simple inspection or to detect by other methods. Unlike computer algorithms that follow a set of instructions, NN learn by mean of examples that will largely determine the quality of the results obtained.

The human nervous system is composed by particular cells called neurons that send signals rapidly through their myelinated projections (axons) with the goal of inhibiting or exciting the neighbor neuron(s) or cell(s). The junctions between neurons are called synapses and the network of connected neurons is responsible for the human body perception of the world stimulus and its feedback. Similarly, NN algorithms consist of a large number of connected units (neurons), typically organized in several layers, with the signal passing from the input neuron layer to the output neuron layer in a feed-forward process. The synapses between neurons are weighted connections and the learning process can be simply described as finding the weights that enable the NN to best capture the trends buried in the data set. Typically, several computational stages are required for an accurate learning process, where each stage updates the network weights. There are many different forms of network architectures, signal propagation or weights transformation. For a detailed description of different types of NN, Schmidhuber provides an overview of deep learning using NN [37. Here, $\mathrm{RBF}$ are used for a non-linear update of the weights of a multi-layer feed-forward NN.

\section{$3 \quad$ Results}

Since the unit of analysis in the present study was the group, and team psychological capital was obtained from team members, it was necessary to aggregate efficacy, hope, resilience and optimism to the team level (the remaining variables were already at the group level). To justify aggregation, the Average Deviation Index $\left(\mathrm{AD}_{M}\right.$ Index) developed by Burke, Finkelstein, and Dusig (1999) was calculated. The average ADM values obtained for efficacy, hope, resilience and optimism were $0.43,0.41,0.45$ and 0.42 , respectively. Since all the 
Table 1. Correlation analysis.

\begin{tabular}{|c|c|c|c|}
\hline & 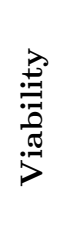 & 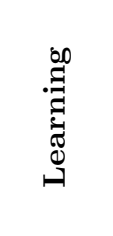 & 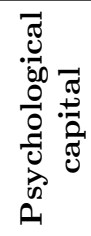 \\
\hline Viability & 1 & $.590^{* *}$ & $.499^{* *}$ \\
\hline Learning & & 1 & $.395^{* *}$ \\
\hline $\begin{array}{c}\text { Psychological } \\
\text { capital }\end{array}$ & & & 1 \\
\hline
\end{tabular}

Table 2. Optimal CV errors for the data set.

\begin{tabular}{cccc}
\hline Multiquadric & Thin Plate & Cubic & Gaussian \\
CV Error & CV Error & CV Error & CV Error \\
\hline 3.84 & 0.64 & 1.38 & 3.11 \\
\hline
\end{tabular}

values were below the upper-limit criterion of 1.01, team members scores were aggregated, with confidence, to the team level.

Table 1 displays the (Pearson) correlation analysis performed to assure that team psychological capital and team learning are correlated with team viability. Significant and positive correlations were found between the independent variables, psychological capital and learning, and team viability. Significant and positive correlation was also found between psychological capital and learning. Despite that, the inclusion of both variables as predictors of team viability is licit since in social sciences correlations of that magnitude are considered of medium-size [5].

Optimal RBF model parameters $\theta_{1}, \ldots, \theta_{n}$ of Eq. 3 were computed by minimizing the CV error using a MATLAB implementation (fminsearch) of a derivative-free optimization algorithm called Nelder-Mead [29. The optimal CV error obtained for the different basis functions tested was used as proxy of their prediction ability [34. Optimal CV errors for the RBF interpolation models obtained by using different basis functions are displayed in Table 2 . Thin plate RBF was 


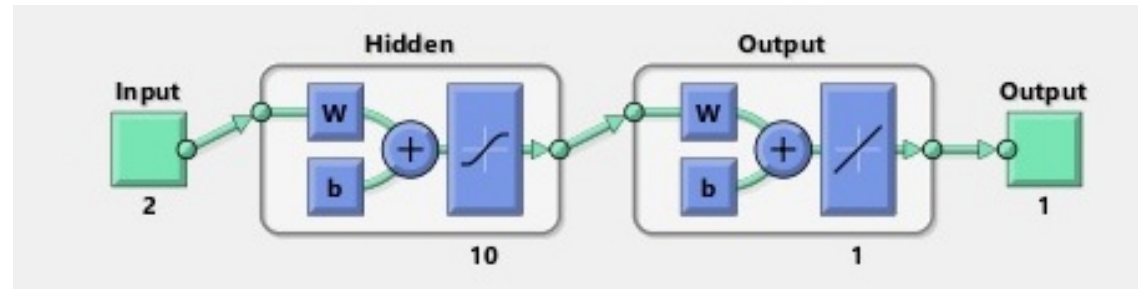

Fig. 1. Neural network architecture.

selected as basis function since the corresponding RBF model presented the lowest CV error.

MATLAB Neural Network Toolbox [28] was used to compute the RBF NN model. The data set was randomly divided into three subsets: training data set - used by the network to adjust its weights during learning/fitting -, validation data set - used to halt training when network's generalization is not improving - and testing data set - to provide an independent measure of the network's prediction ability. The training data set considered $65 \%$ of the entire data set (53 data points - teams), the validation data set 10\% (8) and the training data set $25 \%$ (21). The network architecture considered two inputs (psychological capital and learning), one output (team viability) and 10 neurons in the fitting network's hidden layer as illustrated in Fig. 1. The network was trained/fitted using RBF regression. $R$-values obtained for the three data subsets and the entire data set are displayed in Fig. 2. The predicting ability inherent to the $R$-value (and consequently $R^{2}$-value) obtained for the training set is quite good. Since RBF regression is an interpolation method, the $R^{2}$-value for the data set where the model is fit will be exactly one. Thus, in order to compare the results with the RBF NN model, a Thin Plate RFB model was fit using the data set composed by the training set and the validation set of the RBF NN model. Then, that Thin Plate RBF model was used to predict team viability for the remaining 21 data points corresponding to the testing set of the RBF NN model. Fig. 3 display the prediction of team viability for 21 data points using the RBF NN model and the Thin Plate RBF model. In order to benchmark these results, the following multiple linear regression model, obtained using SPSS, was also fitted and tested considering the exact same fitting and testing data sets:

Team viability $=0.01+0.315 \times($ psychological capital $)+0.465 \times($ learning $)$.

$R^{2}$-values obtained by the linear model, the Thin Plate RBF model and the RBF NN model for the testing data set were $.39, .31$ and .47 , respectively.

Fig. 4 display the relationships between both explanatory variables and team viability, captured by the different models. Data points of the training set and the testing set were added to the plots for a better perception of the scatter in the data. 

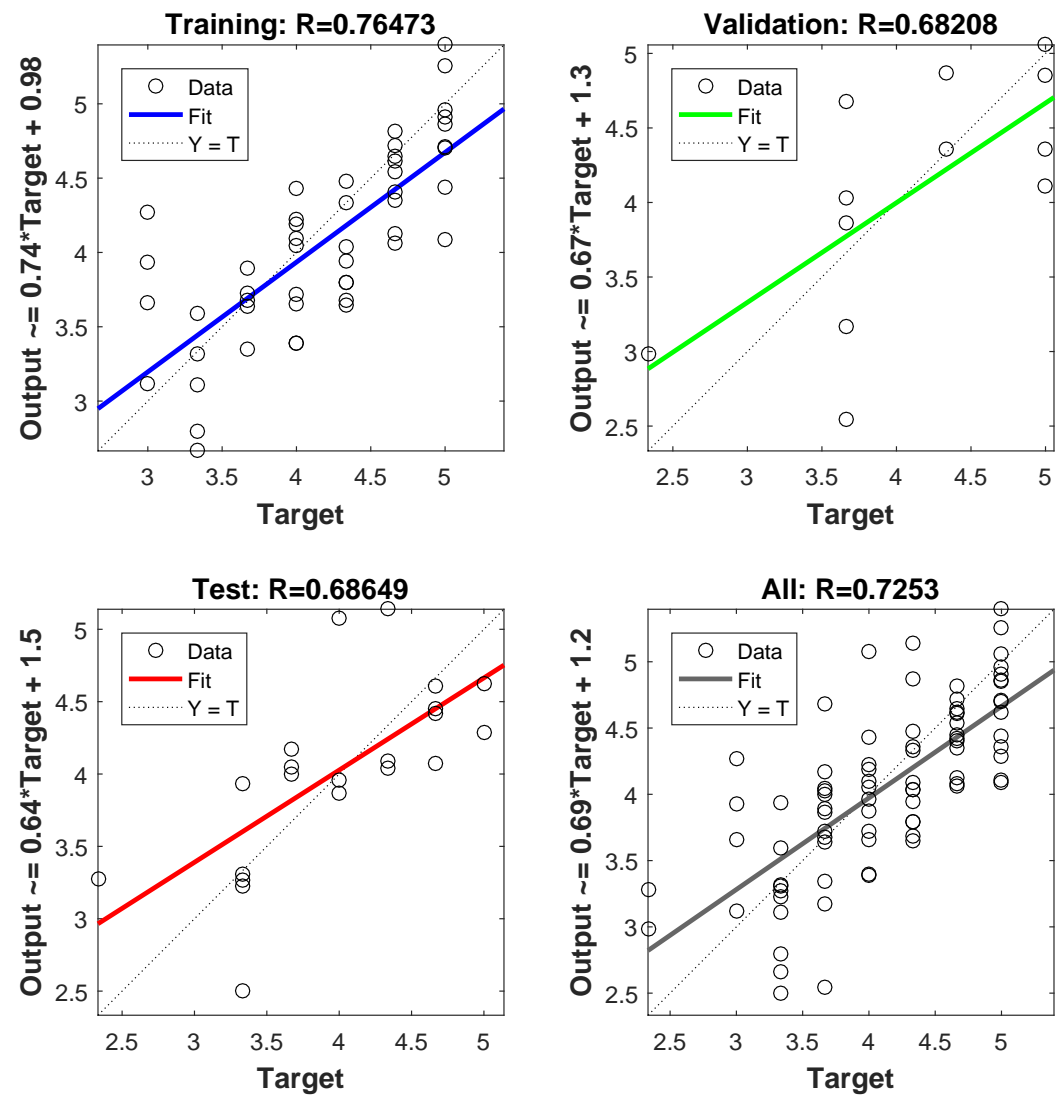

Fig. 2. $R$-values obtained by the RBF NN model for the different subsets considered.

\section{Discussion and Conclusions}

It is possible to find many different models that present similar responses for a given data set. This is also valid for models whose response is $100 \%$ accurate for all data points (interpolation models) as RBF models. However, model's behavior between data points, i.e. trends or relationships between variables, may be completely different for distinct models. A simple way to find the model that present the most reliable trends is to verify which model present the most accurate predictions for new data points.

Prediction results obtained by RBF NN model clearly outperform linear and Thin Plate RBF model's results. That is clear by simple inspection of Fig. 3 or by comparing the $R^{2}$-values obtained for the testing data set. Thus, the trends 

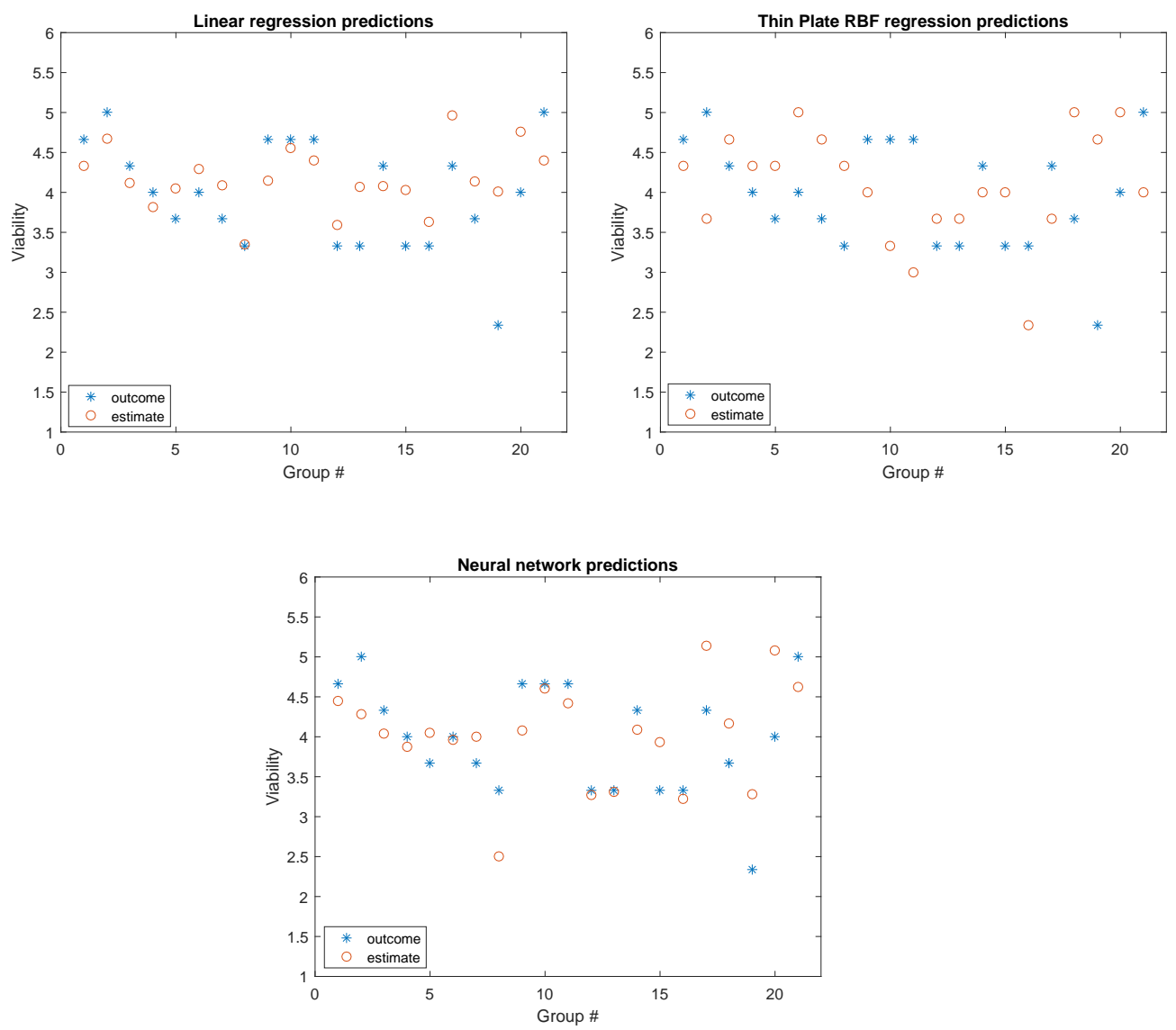

Fig. 3. Prediction of linear, Thin Plate RBF and RBF NN models.

or relationships between team psychological capital, team learning and team viability are more reliable for the RBF NN model. These trends can be easily inspected in Fig. 4. These plots, obtained by MATLAB, are actually dynamic 3D surfaces whose inspection from different angles enable a better understanding of the nonlinear trends. For the RBF NN response surface, an increase in either team psychological capital or team learning leads to an increase in team viability up to a certain threshold where team viability cease to increase. As expected, the linear response is a plane that presents similar trends except that they are linear and thus team viablity always increase for an increase of either team psychological capital or team learning. Finally, although the Thin Plate RBF response surface presents a nonlinear trend similar to the RBF NN response surface, it also presents many peaks (sharp oscilations). This is related to the 

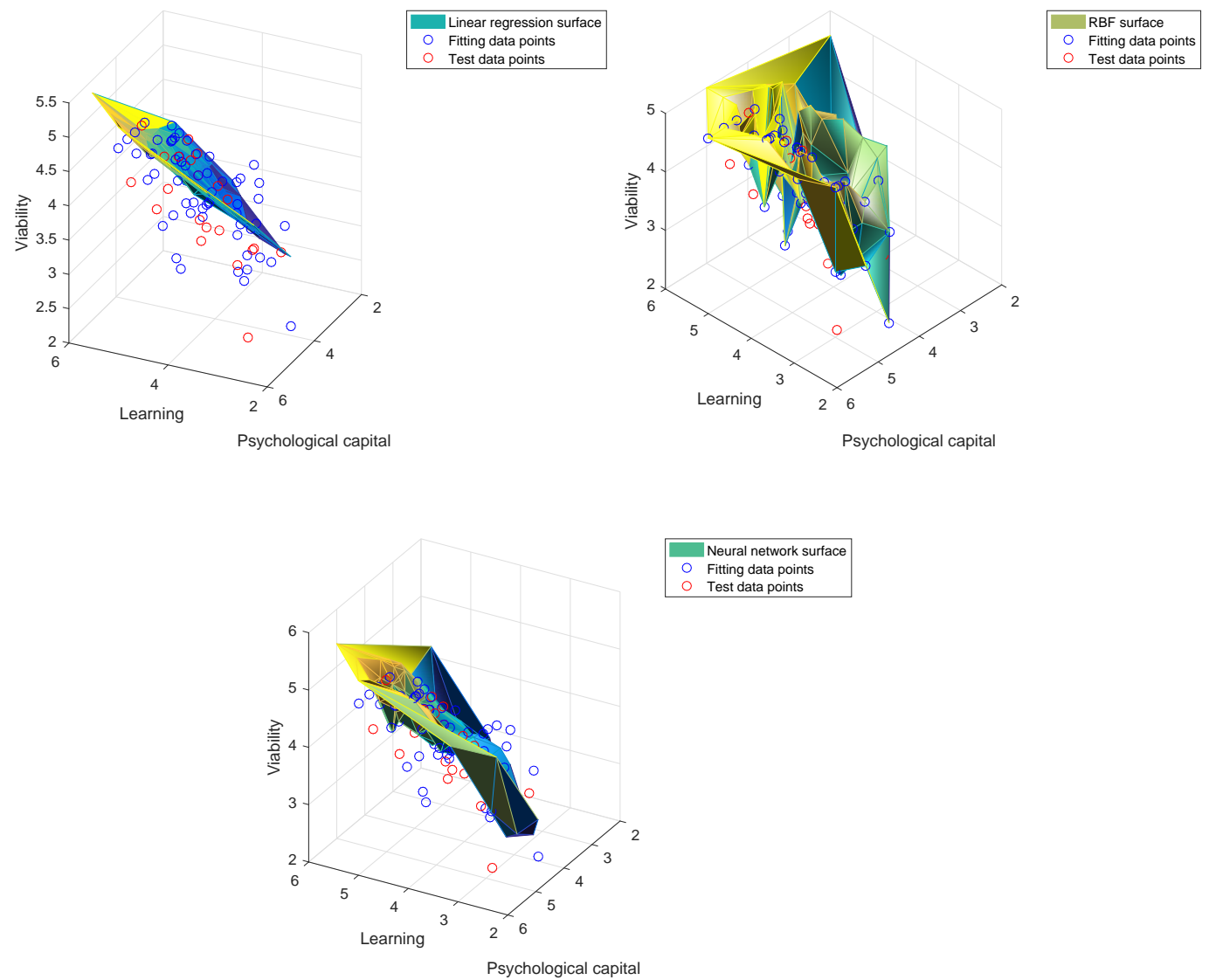

$\circ$ Fitting data points

Test data points

Fig. 4. Three-dimensional surface of linear, thin plate RBF and RBF neural networks models.

fact of RBF models being interpolation models and this particular data set clearly do no favor interpolation type of methods.

The results presented revealed nonlinear patterns between the predictor variables, team psychological capital and team learning, and the criterion variable, team viability. An increasing trend up to a certain threshold is obtained, followed by a deflation for the highest values of the predictors. Thus, our results, which extend the conclusions of previous studies to different samples and variables [13, clearly highlight that more is not always better. Concerning team learning, the present findings are in line with those obtained by Bunderson and Sutcliffe [6] and also Dimas et al. [13], and add to the growing body of knowledge that considers the negative effect on effectiveness of an excessive focus on learning. Hence, the involvement of the team in learning behaviors such as exploring 
new ways of performing the tasks, experimenting alternatives or discussing errors, is positive for team results but when all the resources and energy of the team is focused on that behaviors, goals achievement might suffer threatening the viability of the team. As for team psychological capital, our results extends the findings obtained, at the individual level, by Vancouver et al. [42, and also the remarks made by Veraharen regarding the construct of self-efficacy [44, to the team level and to the broader concept of psychological capital. Our findings highlighted that when the levels of team psychological capital are too high, team viability might suffer, probably because the team became overconfident, neglecting some important aspects of the task and committing more errors, undermining, in consequence, the future of the team.

\section{Acknowledgements}

This work was supported by the Fundação para a Ciência e a Tecnologia (FCT) under project grants UID/MULTI/00308/2013 and POCI-01-0145FEDER-008540.

\section{References}

1. Albuquerque, L. B. Team resilience and team effectiveness: Adaptation of measuring instruments (master thesis). Faculdade de Psicologia e de Ciências da Educação da Universidade de Coimbra, Coimbra (2016)

2. Avey, J. B., Luthans, F., Youssef, C. M.: The additive value of positive psychological capital in predicting work attitudes and behaviors. J. Manage. 36, 430-452 (2010)

3. Aubé, C., Rousseau, V.: Team goal commitment and team effectiveness: The role of task interdependence and supportive behaviors. Group. Dyn-Theor. Res. 9, 189204 (2005)

4. Bandura, A.: Social learning theory. Prentice Hall, Upper Saddle River, NJ (1977)

5. Bryman, A., Cramer, D.: Quantitative data analysis for social scientists (rev. Ed.). Florence, KY, US: Routledge (1994)

6. Bunderson, J.S., Sutcliffe, K.M.: Management Team Learning Orientation and Business Unit Performance. J. Appl. Psychol. 88, 552-560 (2003)

7. Burke, M.J., Finkelstein, L.M., Dusig, M.S.: On average deviation indices for estimating interrater agreement. Organ. Res. Methods 2, 49-68 (1999)

8. Chan, C., Pearson, C., Entrekin, L.: Examining the effects of internal and external team learning on team performance. Team Perform. Manage. 9, 174-181 (2003)

9. Clapp-Smith, R., Vogelgesang, G. R., Avey, J. B.: Authentic Leadership and Positive Psychological Capital: The Mediating Role of Trust at the Group Level of Analysis. J. Leadersh. Organ. Stud. 15, 227-240 (2008)

10. Cohen, S. G., Bailey, D. E.: What makes teams work: Group effectiveness research from the shop floor to the executive suite. J. Manage. 23, 239-290 (1997)

11. Cortina, J. M., Aguinis, H., DeShon, R. P.: Twilight of dawn or of evening? A century of research methods in the Journal of Applied Psychology. To appear in J. Appl. Psychol. (2017) 
12. Decuyper, S., Dochy, F., Bossche, P.V.: Grasping the dynamic complexity of team learning: An integrative model for effective team learning in organizations. Educ. Res. Rev. 5, 111-133 (2010)

13. Dimas, I., Rocha, H., Rebelo, T., Loureno, P.: A nonlinear multicriteria model for team effectiveness. In: Murgante, B. et al. (Eds.) Lecture Notes in Computer Science 9789, pp. 595-609. Springer International Publishing, Switzerland (2016)

14. Edmondson, A.C.: Psychological safety and learning behavior in work teams. Admin. Sci. Quart. 44, 350-383 (1999)

15. Flood, P., Maccurtain, S., West, M. :Effective top management teams: An international perspective. Blackhall Publishing, Dublin (2001)

16. Gully, S. M., Incalcaterra, K. A., Joshi, A., Beauien, M. J.: A meta-analysis of team-efficacy, potency, and performance: Interdependence and level of analysis as moderators of observed relationships. J. Appl. Psychol. 87, 819-832 (2002)

17. Hackman, J.R.: The design of work teams. In: Lorsch, J. (Eds.) Handbook of organizational behavior, pp. 315-342. Prentice-Hall, Englewood Cliffs, NJ (1987)

18. Hackman, J.R.: From causes to conditions in group research. J. Organ. Behav. 33, 428-444 (2012)

19. Hanges, P. J., Lord, R. G., Godfrey, E. G., Raver, J. L.: Modeling nonlinear relationships: Neural networks and catastrophe analysis. In: Rogelberg, S. G., Handbook of research methods in industrial and organizational psychology, pp. 431-455. Blackwell Publishing, Maiden, MA (2004)

20. Heled, E., Somech, A., Waters, L.: Psychological capital as a team phenomenon: Mediating the relationship between learning climate and outcomes at the individual and team levels. J. Posit. Psychol. 9760, 1-12 (2015)

21. Kozlowski, S.W.J., Ilgen, D.R.: Enhancing the Effectiveness of Work Groups and Teams. PSPI 7, 77-124 (2006)

22. Luthans, F., Avey, J. B., Avolio, B. J., Norman, S. M., Combs, G. M.: Psychological capital development: Toward a micro-intervention. J. Organ. Behav. 27, 387-393 (2006)

23. Luthans, F., Norman, S. M., Avolio, B. J., Avey, J. B.: The mediating role of psychological capital in the supportive organizational climate-employee performance relationship. J. Organ. Behav. 29, 219-238 (2008)

24. Luthans, F., Youssef, C. M.: Human, social, and now positive psychological capital management. Organ. Dyn. 33, 143-160 (2004)

25. Luthans, F., Avolio, B. J., Avey, J. B., Norman, S. M.:Positive Psychological Capital: Measurement and Relationship with Performance and Satisfaction. Pers. Psychol. 60, 541-572 (2007)

26. Mathieu, J. E., Heffner, T. S., Goodwin, G. F., Salas, E., Cannon-Bowers, J. A.: The influence of shared mental models on team process and performance. J. Appl. Psychol. 85, 273-283 (2000)

27. Mathe-Soulek, K., Scott-Halsell, S., Kim, S., Krawczyk, M.: Psychological capital in the quick service restaurant industry: A study of unit-level performance. J. Hosp. Tour. Res. 24 (2014)

28. MATLAB 2016a. Natick. The MathWorks Inc., Massachusetts (2016)

29. Nelder, J., Mead, R.: A Simplex Method for Function Minimization. Comput. J., 308-313 (1965)

30. Newman, A., Ucbasaran, D., Zhu, F., Hirst, G.: Psychological capital: A review and synthesis. J. Organ. Behav. 35, s1 (2014)

31. Powell, M.: Radial Basis Function Methods for Interpolation to Functions of Many Variables. HERMIS: Int. J. Computer Maths \& Appl. 3, 1-23 (2002) 
32. Rocha, H., Li, W., Hahn, A.: Principal Component Regression for Fitting Wing Weight Data of Subsonic Transports. J. Aircraft 43, 1925-1936 (2006)

33. Rocha, H.: Model parameter tuning by cross validation and global optimization: application to the wing weight fitting problem. Struct. Multidiscip. Optim. 37, 197-202 (2008)

34. Rocha, H.: On the selection of the most adequate radial basis function. Appl. Math. Model. 33, 1573 - 1583 (2009)

35. Rocha, H., Dias, J.M., Ferreira, B.C., Lopes, M.C.: Selection of intensity modulated radiation therapy treatment beam directions using radial basis functions within a pattern search methods framework. J. Global Optim. 57, 1065-1089 (2013)

36. Rocha, H., Dias, J.M., Ferreira, B.C., Lopes, M.C.: Beam angle optimization for intensity-modulated radiation therapy using a guided pattern search method. Phys. Med. Biol. 58, 2939 (2013)

37. Schmidhuber, J.: Deep learning in neural networks: An overview. Neural Netw. 61, 85-117 (2015)

38. Stajkovic, A. D., Luthans, F.: Self-efficacy and work-related performance: A meta analysis. Psychological Bulletin 44, 580-590 (1998)

39. Sundstrom, E., De Meuse, K., Futrell, D.: Work teams: applications and effectiveness. Am. Psychol. 45, 120-133 (1990)

40. Tu, J.: Cross-validated Multivariate Metamodeling Methods for Physics-based Computer Simulations. Proceedings of the IMAC-XXI (2003)

41. Youssef, C. M., Luthans, F.: Positive Organizational Behavior in the Workplace: The Impact of Hope, Optimism, and Resilience. J. Manage. 33, 774-800 (2007)

42. Vancouver, J.B., Thompson, C.M., Williams, A.A.: The changing signs in the relationships among self-efficacy, personal goals, and performance. J. Appl. Psychol.8, 605-620 (2001)

43. West, B. J., Patera, J. L., Carsten, M. K.: Team level positivity: Investigating positive psychological capacities and team level outcomes. J. Organ. Behav. 30, 249-267 (2009)

44. Verhaeren, T.: Is a strong sense of self-efficacy always beneficial? BUT Philology/Social Sciences 5, 193-200 (2012) 